\title{
Ethics of Scientific Research in the Era of the Fourth Industrial Revolution
}

Gamal Ali El dahshan

Faculty of Education, Menoufia University, Egypt

DOI:

\begin{abstract}
The present paper seeks to review the most important ethics that must be taken into consideration in researching the applications of the Fourth Industrial Revolution and to benefit from those applications in the service of mankind and to avoid the risks that can arise by addressing the following points:

1. What is meant by the ethics of scientific research? What is its importance at the university?

2. What are the most important ethics of scientific research and Unethical Deviations?

3. What is meant by the Fourth Industrial Revolution and what are its characteristics? What are the ethical dilemmas for the applications of the techniques of the Fourth Industrial Revolution?

4. Moral dilemmas for the applications of the techniques of the Fourth Industrial Revolution

5. Global interest in the ethics of the Fourth Industrial Revolution and artificial intelligence to confront its moral dilemmas.
\end{abstract}

Keywords: Ethics of scientific research, The fourth industrial revolution, Artificial intelligence, Moral dilemmas.

\section{Introduction}

The ethics of scientific research is an important input to achieve the quality of scientific research and improve its outputs.

We are living in the midst of a new industrial revolution. This revolution is different from the past three in terms of speed, scope and impact. It is a digital revolution characterized by a combination of technology that affects every aspect of life; how we work and how we live. It has contributed and will contribute to creating threats and opportunities.

If the industrial revolution has made incredible progress and created inventions that we could not have made possible, computers 
and robots can learn how to improve their performance and even make decisions - of course, by an algorithm and without an individual conscience. But that does not deter us from asking some questions. Can a machine think? What are the capabilities of artificial intelligence at this stage of its development? How autonomous is it? What about human control in all of that?

In fact, all revolutions face many challenges that differ in quality, size and the way to face them. The most prominent challenges facing the industrial revolution and its technologies are the ethical ones. Its results and applications are those challenges that were complicated. Some described it as a moral dilemma.

Some of the applications of the Fourth Industrial Revolution have come into question: the collection of data in violation of private life, facial recognition algorithms that are supposed to be used to identify aggressive or racial bias, unmanned warplanes, lethal autonomous weapons, self-driving vehicles, and many other ethical problems. Their gravity will worsen in the future.

While scientific research is advancing very fast on the technical aspects of the Fourth Industrial Revolution, we find it faltering when it comes to its ethical aspects. It is true that many researchers are expressing concern, and that some countries have begun to think seriously about this issue, but to date there is no legal framework to guide future research at the global level. In this sense, said Audrey Azoulay, Director-General of UNESCO, it is our responsibility to conduct a global and enlightened dialogue so that we can enter this new age with open eyes, without sacrificing our values, and until we can reach a common ground of ethical principles."

In order for the Fourth Industrial Revolution to develop responsibly, it is necessary to establish an international mechanism that regulates its standards. Although the various social, political and economic institutions in our countries are increasingly adopting artificial intelligence techniques, they have not yet clearly identified the consequences of their use. We should not be fascinated by the enormous opportunities and potentials of AI applications and lose sight of the legal and ethical challenges that regulate its systems and applications, ensuring that they are interpretable, safe, useful, fair, controllable, and directed to the common good and human wellbeing.

Some applications of the Fourth Industrial Revolution have become subject of doubt, fear, and concern about the destruction of human life. This raises many ethical and legal problems. The benefits that the Fourth Industrial Revolution can bring to the benefit of 
humanity are offset by the negatives that all societies will suffer from.

Based on this there are dozens of articles about the need for ethics and laws governing the applications of the Fourth Industrial Revolution, such as robots. Will robots control us? Is it possible to get out of the control of its maker? Is it possible to hurt a person by making a wrong decision? To protect mankind from these possibilities, the decisions and actions of robots must be controlled in the future by guiding them with laws, concepts, principles and values.

As we entered the era of machines, we became in need of documented laws to be a world norm. We must stress the importance of ethics, as well as popular terms such as disruption. Last year the American University of Carnegie Mellon University announced the opening of a new center for the study of artificial intelligence. During the term of President Barack Obama, the White House published a paper on the same topic. Then tech giants, including Facebook and Google, announced a partnership to chart a moral structure for artificial intelligence.

The present paper seeks to review the most important ethics that must be taken into consideration in researching the applications of the Fourth Industrial Revolution and to benefit from those applications in the service of mankind and to avoid the risks that can arise by addressing the following points:

1. What is meant by the ethics of scientific research? What is its importance at the university?

2. What are the most important ethics of scientific research and Unethical Deviations?

3. What is meant by the Fourth Industrial Revolution and what are its characteristics? What are the ethical dilemmas for the applications of the techniques of the Fourth Industrial Revolution?

4. Moral dilemmas for the applications of the techniques of the Fourth Industrial Revolution.

5. Global interest in the ethics of the Fourth Industrial Revolution and artificial intelligence to confront its moral dilemmas.

First: The meaning of the ethics of scientific research and its benefits?

\section{What is Ethics?}

Ethics means: To distinguish between right and wrong، then do the right thing, I wish it was that, but the real problem "You are sometimes required to choose between two actions that seem both right and you are confused. What to do?" There are many examples:

What do we mean by Ethics of scientific research? 
Research ethics are concerned with the responsibility of researchers to be honest and respectful to all individuals who are affected by their research studies or their reports of the results.

Research Ethics is a world-wide set of principles governing the way any research involving interaction between the researcher and other humans or data relating to humans, is designed, managed and conducted.

Research ethics committees have an important role to play in ensuring the ethical standards and scientific merit of research involving human subjects. ... Firstly, and most importantly, the ethics committee must ensure that the rights of research participants are protected.

In general, the ethics of scientific research require respecting the rights and opinions of others, whether they are research colleagues, participants in conducting research, or those targeted by the research.

The benefits of ethical commitment at the university:

There are many benefits for having ethical committees at universities. The benefits are as follows:

1. Considering ethics contributes to the improvement of society as a whole so that unjust practices are decreased, equal opportunities for people become available, and tasks are carried out by the most efficient ones. Committing to ethics also leads to the efficient use of limited resources, and the path is gradually blocked in the face of parasites and swindlers, and gradually expands to the hard-working. All this and more is achieved if everyone is committed to morality.

2. Commitment to work ethics contributes to social satisfaction among the majority of people as a result of fair dealing, transactions, contracts, assigning business, distributing wealth, linking income with effort, etc.

3. Work ethic supports the environment conducive to team spirit and increased productivity, which benefits the individual, the organization and society.

4. Managing work ethics efficiently so that employees and professors could feel selfconfident at work. This reduces anxiety and stress and achieves more stability and psychological comfort.

5. The moral commitment in the organization secures it against risks to a large extent, where there is a commitment to legitimacy, away from irregularities or a crime, adhering to law as it is more or less only a moral value.

6. Commitment to business ethics supports a number of other important programs such 
as human development programs, total quality programs, and strategic planning programs, all of which support the organization and its development and success.

7. Adherence to strict ethical conventions drives dealers to resort to ethically committed entities, thus good or correct practice succeeds in driving bad practice out of business.

8. The existence of a code of ethics for the profession or organization serves as a guide for everyone not only in their actions, but also when disagreements arise about which behavior to follow.

\section{Second: Ethical Considerations for Scientific}

\section{Research and Unethical Deviations?}

There are differences between the opinions of scientists and researchers about the most important elements that constitute the basic ethics of scientific research and the immoral elements that must be avoided. However, there is almost a consensus among them on a set of general and basic ethics that can be addressed as follows:

1. (Truthfulness): Your research results should be honest, and you have to be honest in what you transmit to others. Do not use unreliable resources to complete any missing information. Do not try to enter data based on the results of theories, or other persons.

2. Experience (Expertise): Your research should be relevant to your level of experience and training, first prepare the initial work and then try to understand the theory accurately before applying the concepts or procedures. Experts in your field of study could be the best assistant for you to choose the things that you should consider.

3. (Safety): Do not expose yourself to any physical or moral risk, and take precautionary preparations when performing your experiments. Do not try to implement research in environments that may be geologically, socially, or chemically hazardous. The safety of the target audience of the search is important as well. Do not make them feel ashamed or expose them to risk in the subject of research.

4. (Trust): Try to build a relationship of trust with those who work with you, so you get greater cooperation and the results are more accurate. Do not exploit the trust of the participants of your study.

5. (Informed Consent): Always make sure you have the prior approval of those whom you would like to work with during the period of the research. They should know 
that they represent the sample of your study. For example, if you need to get access to the property of others, you have to get their consent to it, failure to initial planning of your research may oblige you to search for other sites and start again.

6. (Withdrawal): People have the right to withdraw from the study at any time. Always remember that the participants are often volunteers and should be treated with respect and realize that the time they spend in your research can be used in a more profitable and useful task. Therefore, you should expect the withdrawal of some of the participants. That is why it would be better to start your research with a large possible number of individuals, so that you can continue with a group large enough to make sure that your research results are meaningful.

7. (Digital Recording): Do not record voices and videos or take photos without the consent of the target audience of the research, and get prior approval before the start of any recording. Do not try to use cameras or hidden tapes for recording sounds and movements of the participants, and you must be aware that the request for approval after the recording is unacceptable.
8. (Feedback): If you can give feedback to the target of the research, then do, you may not be able to provide participants with full report, but you may give them a summary or some phrases. Recommendations may be important to participants. It is very important to offer them the photos and their recorded voices and the printed texts of what they said before publishing, so that they are not bodily or mentally harmed. Make sure you always take prior approval before publishing.

9. (False Hope): Do not make the target think through your questions that things will change because of your research or project, and do not give promises outside the scope of your research, position or influence.

10. Vulnerability: Some participants may be more likely subject to a feeling of defeatism because of the age factor, disease or inability to understand or express their thoughts. You must take into account their feelings.

11. Exploiting positions (Exploitation): Do not exploit situations to serve your research, do not explain what you notice or what others say indirectly to serve your research.

12. Confidential information (Anonymity): You have to protect the identity of the target at all times. Do not give names or hints that could lead to the disclosure of 
their true identity, and this can be achieved by converting names to numbers or symbols with making sure that everything related to the identity of the target is completely destroyed.

13. (Animal Rights): If your studies are related to animals, there are ethical considerations in this regard. You should care about the animal and feel the extent of pain and discomfort he has. This is in line with the requirements of the objectives of any study or research you do. You should be looking for advice from teacher supervisor and the expert in the field of research conducted before starting any study that requires the presence of animals, whether in the laboratory or in the field of study.

\section{Principles of Ethical Research:}

There are a set of ethical standards that must be observed in field research as follows:

1. Research should be designed, reviewed and undertaken to ensure integrity and quality.

2. Research staff and subjects must be informed fully about the purpose, methods and intended possible uses of research, what participation in research entails and what risks, if any, are involved.

3. Confidentiality of information supplied by research subjects \& anonymity of respondents must be respected
4. Research participants must participate in a voluntary way, free from any obligation.

5. Harm to research participants must be avoided.

6. Independence of research must be clear, and any conflicts of interests or partiality must be explicit

\section{Contraband in scientific research (Unethical}

\section{Deviations):}

If we have talked in the previous paragraphs about a set of ethical standards, it must be taken into consideration to carry out scientific theoretical and field research, then we complete this by reviewing the most important prohibitions or ethical deviations that researchers must avoid while conducting their research, as follows:

1. Publishing the same paper in two different journals without informing editors.

2. Submitting the same paper to different journals without informing the editors.

3. Not informing coauthors of your intent to file a patent in order to pretend that you are the sole inventor.

4. Including a colleague as an author on a paper in return for a favor even though the colleague did not make a serious contribution to the paper. 
5. Discussing with your colleagues confidential data from a paper that you are reviewing for a journal.

6. Trimming outliers from a data set without discussing your reasons in paper.

7. Using an inappropriate statistical technique in order to enhance significance of research.

8. Bypassing peer review process and announcing your results through a Press Conference without giving peers adequate information to review your work.

9. Conducting a Review of Literature that fails to acknowledge the contributions of other people in the field or relevant prior work.

10. Exaggerating concepts to get a study grant in order to convince reviewers that your project will make a significant contribution to the field.

11. Stretching the truth on a Job Application or Curriculum Vitae

12. Giving the same research project to two graduate students in order to see who can do it faster.

13. Overworking, neglecting, or exploiting graduate or post-graduate students.

14. Failing to keep good research records.
15. Failing to maintain research data for a reasonable period of time.

16. Making humiliating comments and personal attacks in your review of author's submission.

17. Making significant deviations from the research protocol approved by your institution's Animal Care or Committee or Institutional Review Board for Human Subjects Research without telling the Committee or the Board.

18. Not reporting an Adverse Events or side effects in a human research experiment.

19. Wasting animals in research.

20. Exposing Students and Staff to biological risks in violation of your institution's Biosafety Rules.

21. Rejecting a manuscript for publication without even reading it.

22. Damaging someone's work.

23. Stealing supplies, books, or data.

24. Rigging an experiment so you know how it will turn out.

Third: On the Fourth Industrial Revolution, and its characteristics and the ethical dilemmas for the applications of the techniques of the Fourth Industrial Revolution.

The Fourth Industrial Revolution is the current and developing environment in which disruptive technologies and trends 
such as the Internet of Things (IoT), robotics, virtual reality (VR) and artificial intelligence (AI) are changing the way we live and work.

The Fourth Industrial Revolution is a way of describing the blurring of boundaries between the physical, digital, and biological worlds. It's a fusion of advances in artificial intelligence $(\mathrm{AI})$, robotics, The Internet of Things (IOT), 3D printing, genetic engineering, quantum computing, and other technologies. It's the collective force behind many products and services that are fast becoming indispensable to modern life. Think GPS systems that suggest the fastest route to a destination, voice-activated virtual assistants such as Apple's Siri, personalized Netflix recommendations, and Facebook's ability to recognize your face and tag you in a friend's photo.

The easiest way to understand the Fourth Industrial Revolution is to focus on the technologies driving it. These include the following:

Artificial intelligence (AI): describes computers that can "think" like humans, recognizing complex patterns, processing information, drawing conclusions, and making recommendations. AI is used in many ways, from spotting patterns in huge piles of unstructured data to powering the autocorrect on your phone.

Blockchain: is a secure, decentralized, and transparent way of recording and sharing data, with no need to rely on third-party intermediaries. The digital currency Bitcoin is the best known blockchain application. However, the technology can be used in other ways, including making supply chains traceable, securing sensitive medical data anonymously, and combating voter fraud.

New computational technologies are making computers smarter. They enable computers to process vast amounts of data faster than ever before, while the advent of the "cloud" has allowed businesses to safely store and access their information from anywhere with internet access, at any time. Quantum computing technologies now in development will eventually make computers millions of times more powerful. These computers will have the potential to supercharge AI, create highly complex data models in seconds, and speed up the discovery of new materials.

Virtual reality (VR): offers immersive digital experiences (using a VR headset) that simulate the real world, while augmented reality merges the digital and physical worlds. Examples include L'Oréal's makeup app, which allows users to digitally 
experiment with makeup products before buying them, and the Google Translate phone app, which allows users to scan and instantly translate street signs, menus, and other text.

\section{Biotechnology harnesses cellular and} bimolecular processes: to develop new technologies and products for a range of uses, including developing new pharmaceuticals and materials, more efficient industrial manufacturing processes, and cleaner, more efficient energy sources. Researchers in Stockholm, for example, are working on what is being touted as the strongest biomaterial ever produced.

Robotics: refers to the design, manufacture, and use of robots for personal and commercial use. While we're yet to see robot assistants in every home, technological advances have made robots increasingly complex and sophisticated. They are used in fields as wide-ranging as manufacturing, health and safety, and human assistance.

3D printing: allows manufacturing businesses to print their own parts, with less tooling, at a lower cost, and faster than via traditional processes. Plus, designs can be customized to ensure a perfect fit.

Innovative materials, including plastics, metal alloys, and biomaterials, promise to shake up sectors including manufacturing, renewable energy, construction, and healthcare.

Fourthly: Moral dilemmas for the applications of the techniques of the fourth industrial revolution:

The field of information technology ethics is a new subject in the social and human sciences. Many of the new issues arising from this field were not previously known. The problem lies in the nature of dealing with these ethical issues because of following the method and method itself in studying and analyzing it and following in most social issues. There should be clarifying of the social effects of different information technology and then applying ethical theories to those effects and their consequences.

Indeed, as soon as we start thinking about the ethical value framework that the techniques of the Fourth Industrial Revolution and artificial intelligence should adopt, build robots according to it, work through it, and gain some moral logic, another very important problem arises regarding the subject of ethics itself and its philosophy.

If artificial intelligence had to operate according to a certain ethical paradigm, what model would it be? Moral paradigms 
and values differ in different cultures, peoples and religions, and even within one people. They may differ from time to time, and from one group to another, not to mention the difference between the individuals themselves.

Our application and development of the systems and techniques of the Fourth Industrial Revolution and "artificial intelligence", ignoring the risks involved in some cases, may lead us to feel remorse for the way in which some of these systems may evolve, as well as not to make room clear in order for the ethical dimension to be considered in this regard

This matter and others will lead to the emergence of what can be called moral dilemmas, which means that we fall into the contradiction between two moral things, one of which requires violating the other, and one of its most prominent manifestations is the following:

1. "Drones": One of the serious repercussions posed by artificial intelligence techniques is the threat of these technologies to the human right to life. This is evident in the case of "independent combat systems" such as "Drones" that carry weapons, or existing combat robots, where the danger here is that these the devices are designed mainly for destruction, so what happens if it falls into the wrong person's hand, or it is penetrated as a result of insufficient or human error in the insurance procedures, and the algorithms that control it were tampered with. Results will be disastrous. The question: How moral is the drone, Drones attacks and the extent of their legitimacy and legality; is there a need for international and moral law to regulate the work of these aircraft?

2. Self-driving Cars: Although selfdriving cars can contribute to providing the comfort people have long sought, increasing safety, reducing traffic congestion, reducing pollution, and reducing accidents and many others, at the same time they raise many ethical problems and dilemmas. Let us imagine this situation: a self-driving car driving inside is a family of four, which starts on a two-lane highway, and the car detects a ball from a distance that jumps to the road in front of it. As soon as the car approached the place, a little boy running down the middle of the road to get his ball back. In a situation like this ... should the car risk the lives of its passengers by sideways (where the roadside meets a steep slope)? Or should 
the car follow its route to ensure the safety of its passengers at the expense of the child's life? This scenario and others present ethical dilemmas that car manufacturers, regulators and supervisors and the buyers of these cars must deal with before granting these cars the full ability to drive themselves.

According to researchers from the Massachusetts Institute of Technology and the University of Oregon as well as the Toulouse School of Economics (cofunded by the French National Center for Scientific Research), "algorithms that control self-driving cars must include ethical principles that guide their decisions in situations where the inevitable occurrence of Harm".

\section{Deep Learning, Machine Learning,} Deep fake: One of the problems of machine learning - it is one of the applications of artificial intelligence the possibility of learning the wrong lessons, and to give a relevant example that shows that machines that learn the language from simulating humans acquire different biases. That is, if the machine evolved through the learning process, it may not be possible to predict how it behaves in the future, nor knowing how to reach its decisions, it follows that the need to find a way to hold the robot accountable for its mistakes, whether moral or legal responsibility, despite the illogical proposition

In addition to the dangerous unethical implications of one of the techniques of machine learning and deep learning, a technique of deep fake that is a new way to trick victims into believing their fake content, and therefore it is another method of fraud used in social engineering, as well as a method. Another methods disinformation will become with time more easy and efficient for all users with the presence of applications that increase progress and development and update their algorithms constantly, and the limit of use will not stop from the limit of artists, celebrities and politicians, but rather the danger of targeting to ordinary people who do not take privacy into account.

This technique, which some have called global horror or chaos technology, can be caused by many risks, including increased chaos and confusion, the possibility of falsifying e-mails and text conversations, loss of confidence in all that can be seen, and the destruction of many lives. Therefore, scientists are 
keen to seek ways to control or reduce the impact of this harmful technology.

4. The Dilemma of privacy and Security: Privacy is one of the biggest individual challenges of information technology. We instinctively understand the importance of privacy, but chasing and sharing our information is a critical part of new relationships. Example: Influencing our private lives, due to the lack of control over our private data in the coming years. Likewise, the revolutions that take place in the field of biotechnology and artificial intelligence, which will redefine the meaning of (human) by changing the boundaries of age, health, perception and capabilities, which will lead us to restore our moral and ethical concepts. The question here is, do human consciences form part of this system? Do ethics have a place?

5. The dilemma of diversity, nondiscrimination and lack of equity or equality: The services provided by Artificial intelligence systems must be available to all, regardless of age, gender, race, or other characteristics. Likewise, systems should not be biased in one category.

The world is witnessing a clear digital divide between those who can use the
Internet because they have the necessary skill and material ability, and those who cannot use the Internet, which is what was called the Digital Divide. That gap has become one of the important issues that occupy the global public opinion. Just like energy and environment issues, not only because the world of knowledge and information affects us and our development to a great degree, but because ambiguity surrounds the knowledge of whether the digital divide is currently narrowing or widening despite international efforts to narrow it at all levels; Because of the enormous development that afflicts the computer and its technologies day after day, and despite the continuous adherence of the middle classes of the information and knowledge world, the mystery still surrounds the knowledge of the narrowness or widening of the digital divide.

Fifth: Global interest in the ethics of the Fourth Industrial Revolution and artificial intelligence to confront its moral dilemmas:

In this context, scholars and researchers presented groups of attempts to overcome these dilemmas by presenting a set of ethical 
values and orientations that should be taken into account in adopting the applications of the Fourth Industrial Revolution techniques in the form of an ethical code directed to those who use these applications, including:

- Isaac Azimov established ethical laws for robots that regulate their rights and duties, as well as determining the basis on which the relationship of the human to the machine and the machine to the human being becomes in harmony, far from any dangers or problems. These laws are:

1. The robot is not permitted to harm a human being or to remain silent about what might cause harm to him

2. A robot must obey human commands unless it conflicts with the first law

3. The robot must maintain its survival if this does not conflict with the first and second laws.

- The report issued recently by the World Summit of Governments in February 2019 in cooperation with "Deloitte", on the impact of artificial intelligence ethics within the government sector, stressed on five ethical considerations that must be dealt with when engaging in artificial intelligence technologies: organizational aspects, governance, legitimacy and non- repudiation, Safety and Security, Social and Economic Impact, Ethics.

- The European Union has also published a set of guidelines on how companies and governments should follow when developing AI applications, the Union held a committee of 52 experts who reached seven requirements that they believe future AI systems must meet. They are as follows:

- Human agency and oversight - AI systems should not bypass human control. People should not be manipulated or coerced by artificial Intelligence systems, and people should be able to intervene or oversee every decision the program makes.

- Technical Accuracy and Safety - That artificial intelligence system be safe and accurate. They should not be easily compromised by external attacks and should be reasonably reliable.

- Privacy and data management - that is, the personal data collected by artificial intelligence systems must be secure and private. It should not be accessible to anyone, and should not be stolen easily.

- Transparency - access to the data and algorithms used to create the AI system is easy, and the decisions that the 
program makes should be understandable. In other words, operators should be able to explain the decisions that AI systems make.

\section{- Diversity, non-discrimination and} fairness - where the services provided by AI systems must be available to all, regardless of age, gender, race or other characteristics. Likewise, systems should not be biased in one category.

- Environmental and societal responsibility - AI systems must be sustainable (that is, they must be environmentally responsible) and "promote positive social change".

- Accountability - AI systems must be auditable and covered by the current protection of whistle-blowers in companies. The negative effects of the systems must be recognized and reported in advance.

The Institute for Future of Life in Boston, USA, set the rules for developing AI applications under the "BENEFICIAL AI" initiative and as an objection to developing drones that choose the target and eliminate it without the intervention of the remote control unit, through a document Consensus signed by a group of world scientists from artificial intelligence scientists, technology pioneers and others, and these principles included 3 general sections that collect 23 principles.

The first section: Research Issues includes the following:

1. Research Objectives: Research on developing AI technologies should not aim to develop non-directed intelligence, but rather useful, useful intelligence.

2. Research funding: Investing in artificial intelligence must be accompanied by funding for research that ensures its beneficial use, including thorny questions in the fields of computer science, economics, law, ethics, and social sciences, such as: How can we make future AI systems strong enough to do the required tasks? Without defect and without penetration? How can we turn to automation to achieve growth and prosperity while ensuring the continuity of the objective of population / employment and the continued source of their income? (One of the thorny issues related to the development of automated and smart technologies is that they replace labor easily and at a lower cost). How can we develop our legal systems to become more accomplished, fair and efficient, in line with the presence of artificial intelligence, and able to deal with the risks associated with it? What 
values should AI have aligned with? What are the laws and moral positions that must be demonstrated?

3. The link between science and politics: There must be a constructive and healthy exchange between researchers and policy makers.

4. Research culture: It should foster a culture of cooperation, trust and transparency between researchers and developers of artificial intelligence technologies.

5. Avoid the race: the various teams developing artificial intelligence technologies must cooperate to avoid exceeding safety standards.

Section Two: Ethics and Values, including the following:

1. Safety: Artificial intelligence systems must be completely safe and secure throughout their working life, always checking this when it is available and possible.

2. Transparency Failure: If an intelligent system causes harm, it should be possible to verify the cause.

3. Transparency. The state of application of a machine: Any participation from an autonomous system in making a judgment must provide a satisfactory and auditable interpretation through a specialized human authority.

4. Responsibility: The designers and builders of advanced artificial intelligence systems are stakeholders in the implications of their use and misuse, bearing the appropriate responsibility and opportunity to shape those effects.

5. Values biasing: The highly independent AI techniques must be designed so that their goals and behaviors are definitely biased towards human values throughout their work.

6. Human values: Smart systems must be designed and managed in a manner consistent with the highest ideals of human values such as dignity, rights, freedoms and cultural diversity.

7. Personal privacy: Human beings have the full right to access and control their data completely, if that data is given to smart systems for the purpose of analysis and use.

8. Freedom and privacy: The application of artificial intelligence systems to personal data must not be withheld from real or perceived personal freedom.

9. Share the benefit: the largest number of people should benefit from smart systems. 
10. Sharing growth and prosperity: The whole of humanity must share the luxury and economic prosperity resulting from the use of artificial intelligence mechanisms.

11. Human Control: Humans choose how and when they want to refer decision making to an intelligent system in order to accomplish the tasks that the user chooses.

12. Not subversive: The power granted to advanced smart systems must respect, develop, and disrupt the approach and civilization upon which a healthy society depends.

13. Smart arms race: The race to develop fully automated armed systems must be avoided.

Section Three: Longer-term Issues, including the following

1. Capacity Caution: For lack of consensus, we should avoid assumptions that anticipate a maximum potential for future AI.

2. Importance: Perhaps artificial intelligence will represent a profound change of the history of life on Earth, and it must be planned to manage the matter with care and proportionate resources
3. Risks: Risks posed by the development of smart systems, especially catastrophic and related to the annihilation of the human race, must become a subject of research and planning to mitigate their results commensurate with their expected effects.

4. Self-development: smart systems designed to develop or manufacture other smart copies in a way that guarantees increased quality or quantity must be subject to a strict safety and control system.

5. The common good: supernatural intelligence should only develop within the framework of serving the most prevalent moral ideals, and to serve the interests of all humanity, and not to serve a specific state or organization.

\section{Conclusion}

In light of all the above, we see the following:

It is imperative that we immediately start to form working teams of experts in the field of science and technology ethics to discuss legal ethical challenges and priorities related to the applications and developments of the Fourth Industrial Revolution, develop ethical and legal frameworks that control and regulate the work of the systems of 
those applications, and ensure that these systems and applications are explainable, safe and fair, ensuring confidence in how to benefit from them legalizing, developing and using them for the well-being of mankind and solving their problems.

Humanity is in urgent need of humanity for an ethical covenant to use the techniques of the Fourth Industrial Revolution, in general, and artificial intelligence, in particular, to regulate its applications in our lives and reduce the negative effects of it, or what is called "ethics and jurisprudence of artificial intelligence."

Attention to the applications of the Fourth Industrial Revolution and its development is necessary for the comfort of mankind and the welfare of its continued prosperity, but avoiding the risks and threats resulting from increased dependence on it is also necessary. This is by establishing a regulatory and ethical mechanism that governs the work of those applications. It also helps develop them and avoid its negatives, specifying functions and tasks and formulating ethical and legal frameworks that guarantee the preservation of basic human rights. Innovation in the field of human-friendly applications should be encouraged, too, setting a value system that governs the relationship between them in an era in which the machine may be superior to humans.

Humanity has become, in light of the gap between how we actually use the techniques of the Fourth Industrial Revolution and what should be used for it, in the need to start studying and identifying ethical and legal issues and societal implications for applications of artificial intelligence in order to manage their risks appropriately to help achieve the positive aspects. Policies, management and organization should be developed in a smart way to ensure that it benefits the community and its members maintaining moral and societal values. "There is no bad technology ... there are bad users."

\section{References}

Al-Okaili, Dalal: Deep fakes: The Global Horror Technology, available at

https://annabaa.org/arabic/informatics/20006 (in Arabic).

Coca-Vila I. Self-driving Cars in Dilemmatic Situations: An Approach Based on the Theory of Justification in Criminal Law. Criminal Law Philosophy [Internet]. 2018 Mar 1 [cited 2018 Oct 5]; 12(1): 59-82. Available from https://link.springer.com/article/10.1007\%2Fs 11 $\underline{572-017-9411-3}$ 
Davies A: How Do Self-Driving Cars See? (And How Do They See Me). Wired 2018 Nov 8 [cited 2018 Nov 11]; Available from https://www.wired.com/story/the-know-it-allshow-do-self-driving-cars-see/

El-Dahshan, Gamal Ali: "Cloud Computing". A working paper presented to the first international forum of the Faculty of Education, Benha University "Technology Applications in Education held on 12-13 February 2017 in the conference hall of the Faculty of Commerce, Banha University. (in Arabic).

El-Dahshan, Gamal Ali: Developing teacher preparation programs to cope with the requirements of the Fourth Industrial Revolution, Educational Journal, Suhag Education - Issue 68, December 2019 (in Arabic).

El-Dahshan, Gamal Ali : Ethics of Scientific Research in the Era of the Fourth Industrial Revolution", Lecture in Lecture in The international workshop on the ethics of scientific research"Ethics in Scientific Research methods, Place at Bayero University Kano (BUK), Nigeria 9 December 2019,

El-Dahshan, Gamal Ali : Roles and responsibilities of Scientific Research Ethics committee Workshop for social, humanities , and law review Ethics, The international workshop on the ethics of scientific research "Ethics in Scientific Research methods, Place at
Bayero University Kano (BUK), Nigeria 9 December 2019

El-Dahshan, Gamal Ali: Humanity needs a code of ethics for AI applications Educational Creativity Magazine - July 2019 Issue Arab Education Association.

El-Dahshan, Gamal Ali : Humanity needs a code of ethics for Applications of the Fourth Industrial Revolution "Artificial intelligence as a model" Research paper presented to The joint scientific symposium between the University of Menoufia and the Naif Arab University for Security Sciences on the ethics of scientific research in the era of the Fourth Industrial Revolution and artificial intelligence Held within the activities of the Fourth Ninth International Scientific Conference of the Faculty of Education, University of Menoufia Entitled "Moral Education in Arab Societies (Reality and Expected)" From 30-31 October 2019 (in Arabic).

El-Dahshan, Gamal Ali: The development of digital intelligence DQ our children one of the requirements of life in the digital age International Journal of research in Educational Sciences. (IJRES) Volume (2) No. (4) 2019 (in Arabic).

El-Dahshan, Gamal Ali: Are our educational institutions prepared to deal with the requirements of the Fourth Industrial Revolution? Ebdaa Al Arab Newspaper April 7, 2019 available at 
http://ebdaelarab.com/2019/04/07/\%D9\%87\%D 9\%84\%D9\%85\%D8\%A4\%D8\%B3\%D8\%B3\% D8\%A7\%D8\%AA\%D9\%86\%D8\%A7(in Arabic).

El-Dahshan, Gamal Ali: Employing Internet of Things in Education: Justifications, areas, challenges International Journal of research in Educational Sciences. (IJRES) Volume (2) No. (3) 2019 (in Arabic).

El-Dahshan, Gamal Ali: Ethical dilemmas for the applications of the Fourth Industrial Revolution Working paper submitted to The Fourth Ninth International Scientific Conference of the Faculty of Education, University of Menoufia Entitled Moral Education in Arab Societies (Reality and Expected), 10-31/ 10/2019 (in Arabic).

Grace Shao : What 'deep fakes' are and how they may be dangerous available at https://www.cnbc.com/2019/10/14/what-isdeepfake-and-how-it-might-be-dangerous.html Healthcare, artificial intelligence and the Fourth Industrial Revolution: Ethical, social and legal considerations Available from https://www.researchgate.net/publication/32938 9882 Healthcare Artificial_Intelligence and th e Fourth Industrial Revolution Ethical Social and Legal Considerations South African Jou rnal of Bioethics and Law $2018 \quad 11293$

John Hooker, Tae Wan Kim: Ethical Implications of the Fourth Industrial Revolution for Business and Society Business Publication date: 7 June 2019 Available from https://www.emerald.com/insight/content/doi/10 $.1108 / \mathrm{S} 2514-175920190000003002 / \mathrm{full} / \mathrm{html}$

Judy E. Stern, and Deni Elliott: The Ethics of Scientific Research Published by University Press of New England, Hanover, 1997.

Larry Greenmailer: Ethical dilemmas on the road to self-driving cars is available at https://www.scientificamerican.com/arabic/articl es/news/driverless-cars-will-face-moraldilemmas/

Ludovic Arnold, Sebastian Rebecchi1, Sylvain Chevalier, Hélène Paugam-Moisy: An Introduction to Deep Learning European Symposium on Artificial Neural Networks, Computational Intelligence and Machine Learning. Bruges (Belgium), 27-29 April 2011, Available from http://www.i6doc.com/en/livre/?GCOI=2800110 0817300.

Mildred Solomon: The 4 big ethical questions of the Fourth Industrial Revolution Available from

https://www.weforum.org/agenda/2016/10/howcan-we-enjoy-the-benefits-of-the-fourthindustrial-revolution-while-minimizing-its-risks/ NRSPP Australia: Human Error in Road Accidents [Internet]. [Cited 2018 Nov 11]. Available from 
https://www.nrspp.org.au/resources/humanerror-in-road-accidents/

Nyholm, S., and J. Smids (2016), “The Ethics of Accident-Algorithms for Self-Driving Cars: An Applied Trolley Problem?" Ethical Theory and Moral Practice 19(5): 1275-1289.

Raslan, Ahmad : Azimov did it before them: ethical principles for a future safe artificial intelligence available at https://www.ida2at.com/asilomar-ai-principles/ (in Arabic).

Safia Mahomed : Healthcare, Artificial Intelligence and the Fourth Industrial Revolution: Ethical, Social and Legal Considerations. South African Journal of Bioethics and Law 2018; 11(2):93. https://www.researchgate.net/publication/32938 9882 Healthcare Artificial Intelligence and th e Fourth Industrial Revolution Ethical Social and Legal Considerations South African Jou rnal_of_Bioethics_and_Law_2018_11293

Salama Qualities, Khalil Abu Koura: Studies of Challenges and the Ethics of the Robotics Era Strategic Studies - Emirates Center for Strategic Studies and Research - Abu Dhabi - UAE 2014.

Schwab Klaus : The Fourth Industrial Revolution: what it means, how to respond: Available from: https://www.weforum.org/agenda/2016/01/the- fourth-industrial-revolution-what-it-means-andhow-to-respond/

Taher, Gamal: Presentation on theme: "Medical Research Ethics "Presentation transcript: Available from:

https://slideplayer.com/slide/14279660/ (in Arabic).

The Franklin Institute: he Science of SelfDriving Cars 2016 [cited 2018 Nov 11]. Available from: https://www.fi.edu/science-ofselfdriving-cars

Van Loon, R.J., and M.H. Martens (2015), "Automated Driving and its Effect on the Safety Ecosystem: How do Compatibility Issues Affect the Transition Period?'” Procedia Manufacturing 3: $3280-3285$

Wattfa, Ali Asaad: The Fourth Industrial Revolution is available at http://www.alnoor.se/author.asp?id=7289 (in Arabic).

What are the main disadvantages of Self-driving cars? [Internet]. Global Auto Transportation. 2017 [cited 2018 Nov 11]. Available from: http://www.globalautotransportation.com/ main-disadvantages-self-driving-cars/

What is the fourth industrial revolution: Available from: https://www.salesforce.com/blog/2018/12/whatis-the-fourth-industrial-revolution-4IR.html (in Arabic). 
WHO | Global status report on road safety 2015

[Internet]. WHO. [cited 2018 Nov 11]. Available

from: http://www.who.int/violence injury preve

ntion/road safety status/2015/en/ 